\title{
Reações maternas às emoções negativas dos filhos e autorregulação emocional infantil
}

\author{
Paula Kleize Costa Sales \\ Patrícia Alvarenga
}

\section{RESUMO}

O desenvolvimento da autorregulação emocional acontece na relação com cuidadores primários. Este estudo investigou as relações entre as reações maternas às emoções negativas dos filhos e a autorregulação emocional infantil. Participaram 33 mães e 30 filhos com idade entre seis e sete anos. As reações maternas às emoções negativas dos filhos foram avaliadas por uma escala (CCNES). A autorregulação emocional infantil foi avaliada por uma escala (ERC) e por uma entrevista respondida pelas crianças. O agrupamento de reações maternas não apoiadoras das expressões emocionais infantis correlacionou-se negativamente com a autorregulação infantil informada pela mãe e positivamente com as estratégias de fuga relatadas pela criança. Os achados apoiam a hipótese sobre relações bidirecionais entre as duas variáveis. Reações não apoiadoras estimulam na criança o uso de estratégias de fuga consistentes com o modelo materno, gerando um ciclo interativo em que mãe e criança reforçam reciprocamente a evitação do contato com emoções negativas e prejudicam o desenvolvimento da autorregulação emocional infantil.

Palavras-chave: autorregulação emocional infantil; socialização emocional; reações maternas; estratégias de autorrregulação emocional.

\section{ABSTRACT Maternal reactions to children's negative emotions and children's emotional
self-regulation}

The development of emotional self-regulation occurs in the relationship with primary caregivers. This study investigated relations between maternal reactions to children's negative emotions and children's emotional self-regulation. Thirty-three mothers of children aged 6 to 7 years old and thirty children participated in the study. Mothers' reactions to children's negative emotions were evaluated with a scale (CCNES). Children's emotional self-regulation was evaluated with a scale (ERC) and an interview answered by children. The grouping of unsupportive reactions to children's emotional expression was negatively correlated with children's emotional self-regulation informed by the mother and positively correlated with scape strategies reported by children. The findings support the hypothesis about bidirectional relations between the two variables. Unsupportive reactions stimulate the child to use scape strategies which are consistent with the maternal model, generating an interactive cycle in which mother and child mutually reinforce the avoidance of contact with negative emotions and impair the development of children's emotional self-regulation.

Keywords: children's emotional self-regulation; emotion socialization; maternal reactions; emotional self-regulation strategies.

As emoções são fenômenos multidimensionais e complexos, mas podem ser definidas basicamente como um conjunto de reações fisiológicas que surge como resposta a eventos ambientais ou subjetivos (Rocha et al., 2017; Verma \& Tiwary, 2014) durante um breve intervalo de tempo, desencadeando uma propensão para agir (Frijda, 2008). 0 manejo da experiência

\section{Sobre os autores}

P. K. C.S http://orcid.org/0000-00019079-4956

Universidade Federal da BahiaSalvador, BA

kleize@hotmail.com

P. A.

https://orcid.org/0000-0002

5890-9896

Universidade Federal da Bahia-

Salvador, BA

palvarenga66@gmail.com

\section{Direitos Autorais}

Este é um artigo de acesso aberto e pode ser reproduzido livremente, distribuído, transmitido ou modificado, por qualquer pessoa desde que usado sem fins comerciais. 0 trabalho é disponibilizado sob a licença Creative Commons CC-BY-NC.

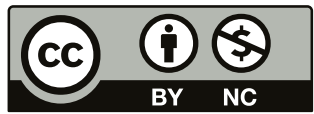




\section{M." INTERACÃO EM PSICOLOGIA}

emocional e da propensão para agir a ela vinculado têm sido investigados por meio do conceito da autorregulação emocional, um conjunto de habilidades que permite aos indivíduos modular a experiência e a expressão de emoções positivas e negativas (Bridges et al., 2004).

$\mathrm{Na}$ infância, a autorregulação emocional repercute nos domínios socioemocional e cognitivo do desenvolvimento, influenciando o ajustamento psicossocial da criança (Jaramillo et al., 2017; Rawana et al., 2014; Zeman et al., 2006). A inabilidade para autorregular as emoções pode gerar diversos prejuízos, como, por exemplo, o surgimento de problemas emocionais e comportamentais (Zeman et al., 2006). Nessa etapa do desenvolvimento, a capacidade de reconhecer e nomear as emoções e de administrar sua intensidade, especialmente no caso de emoções negativas, se ampliam de forma marcante (Rawana et al., 2014; Sabatier et al., 2017; Whitebread \& Basilio, 2012). Diferentes classificações de estratégias de autorregulação emocional usadas pelas crianças podem ser encontradas na literatura. De modo geral, os autores separam estratégias que favorecem a adaptação da criança a diferentes situações e a competência emocional (Carthy et al., 2010; Dunsmore et al., 2012; Meyer et al., 2014) de estratégias que podem obstruir o desenvolvimento emocional e eventualmente ocasionar transtornos mentais (Aldao et al., 2010; Dunsmore et al., 2012; Zeman et al., 2006). No presente estudo, o primeiro conjunto será chamado de "estratégias de enfrentamento" e o segundo grupo será chamado de "estratégias de fuga". Estratégias de enfrentamento estão relacionadas a melhores relações interpessoais (Dunsmore et al., 2012) e a menor intensidade da experiência subjetiva de emoções negativas (Carthy et al., 2010; Meyer et al., 2014). Exemplos de estratégias desse grupo são aquelas focadas no problema que gerou a emoção, na própria emoção ou no direcionamento da atenção e a busca por apoio social (Meyer et al., 2014). As estratégias de fuga associam-se a quadros de ansiedade, depressão, distúrbios alimentares, uso de substâncias (Aldao et al., 2010) e a maior reatividade emocional (Dunsmore et al., 2012; Zeman et al., 2006). Exemplos de estratégias desse tipo são expressar a raiva de modo exacerbado, agredir os outros e tentar evitar ou fugir de um evento que induz emoções negativas frequentemente, ao invés de enfrentar a situação (Zeman et al., 2006).

A aprendizagem de estratégias de autorregulação emocional tem origem na interação da criança com seus cuidadores primários. 0 modelo teórico de Socialização Emocional (Eisenberg et al., 1998) descreve três tipos de contextos em que a criança experimenta oportunidades de aprender sobre suas emoções e as emoções das outras pessoas: as conversas sobre emoções, as reações dos pais às próprias emoções e as reações dos pais às emoções expressas pela criança. De acordo com um estudo de revisão, as investigações acerca da socialização das emoções têm sido conduzidas majoritaria- mente com famílias americanas europeias de classe média (Morelen \& Thomassin, 2013).

Considerando as reações que os pais podem adotar diante da expressão de emoções negativas dos filhos, Eisenberg et al. (1998) e Fabes et al. (2002) propuseram uma tipologia que as classifica nas categorias de reações apoiadoras e reações não apoiadoras da experiência emocional. As reações apoiadoras caracterizam-se por práticas que ajudam a criança a compreender melhor a situação que produziu a emoção e os resultados decorrentes da sua expressividade emocional. Assim, pais que reagem de forma apoiadora ajudam os filhos a se sentirem mais confortáveis e a avaliar relações entre eventos antecedentes e consequentes associados à expressão das emoções (Lins \& Alvarenga, 2018). Esse conjunto de reações se divide em três categorias: a) reações centradas nas emoções, por meio das quais os pais ajudam a criança a encontrar estratégias para se sentir melhor; b) reações centradas no problema, que ocorrem quando os pais focam no que desencadeou as emoções, ajudando a criança a resolver o problema associado à manifestação emocional e c) reações incentivadoras, que se verificam quando os pais acolhem a expressão das emoções negativas dos filhos (Eisenberg et al., 1998; Fabes et al., 2002). Vários estudos correlacionais têm fornecido evidências a respeito das relações entre as reações apoiadoras dos pais e a autorregulação emocional infantil (Meyer et al., 2014; Mirabile, 2015; Han et al., 2015). Adicionalmente, um estudo longitudinal investigou a autorregulação emocional de crianças com e sem sintomas de TDAH e as reações de seus pais e confirmou o poder preditivo das reações apoiadoras (Breaux et al., 2017). Em conjunto, esses achados apoiam a noção de que as reações apoiadoras dos pais às emoções dos filhos facilitam a autorregulação das emoções por parte da criança. É possível também que crianças com maior capacidade de gerenciar a própria experiência emocional evoquem nos pais reações apoiadoras com mais frequência. Morelen e Suveg (2012) examinaram a influência recíproca entre as reações das mães e a autorregulação emocional nos filhos em intervalos de cinco minutos de discussão da díade. Os resultados revelaram que respostas adaptativas da criança às suas próprias emoções eram seguidas mais frequentemente por reações apoiadoras das mães.

Por outro lado, as reações não apoiadoras dos pais são aquelas que punem e invalidam a expressão de emoções negativas dos filhos. Ao reagir desse modo à expressão emocional da criança, os pais não facilitam a aprendizagem da autorregulação, pois tendem a suprimir a manifestação das emoções dos filhos e não transmitem orientações construtivas para lidar com as emoções (Eisenberg et al., 1998; Fabes et al., 2002). Esse grupo de reações é subdividido nas seguintes categorias: a) reações punitivas, que ocorrem quando os pais punem as crianças fisicamente ou verbalmente para 


\section{M." INTERACÃO EM PSICOLOGIA}

controlar sua expressão emocional negativa; b) reações minimizadoras, que podem ser notadas quando os pais minimizam ou desvalorizam as expressões emocionais da criança; c) reações de desconforto, que ocorrem quando os pais se sentem aborrecidos ou desconfortáveis com as emoções negativas da criança (Fabes et al., 2002) e d) reações de indiferença, aquelas que ignoram a expressão de emoções pela criança (Mirabile, 2015).

Vários estudos correlacionais também têm revelado evidências a respeito das relações entre as reações não apoiadoras dos pais e a baixa autorregulação emocional infantil (Miller-Slough et al., 2016; Mirabile, 2015; Shewark \& Blandon, 2015). De modo semelhante ao que foi constatado sobre as reações apoiadoras, o estudo longitudinal de Breaux et al. (2017) também apoiou o poder preditivo das reações não apoiadoras sobre a labilidade emocional. Em conjunto, os resultados dessas investigações indicam que as reações não apoiadoras prejudicam o desenvolvimento da autorregulação emocional na infância. Poucos estudos adotaram delineamentos que possibilitassem examinar o efeito da capacidade de autorregulação emocional da criança sobre as reações maternas. Um exemplo foi a pesquisa de Premo e Kiel (2014) que revelou que o aumento pela busca de contato com as mães por parte dos meninos foi associado à redução das reações maternas não apoiadoras.

É importante destacar, ainda, o fato de que há estudos que não encontraram relações entre as reações não apoiadoras e indicadores de autorregulação emocional das crianças (Han et al., 2015; McCord \& Raval, 2016), e um estudo que, ao contrário do esperado, verificou correlações negativas entre reações apoiadoras e a capacidade de autorregulação emocional infantil (Rogers et al., 2016). Esses estudos indicam que as reações parentais podem apresentar diferentes significados e funções entre as culturas (Han et al., 2015; McCord \& Raval, 2016). Os valores cultivados em determinada cultura, assim como o entendimento das emoções no meio sociocultural mais amplo, influenciam as teorias, objetivos e estratégias de socialização emocional das mães. Essa relação, por conseguinte, influencia o aprendizado da autorregulação emocional das crianças, que deve reincidir sobre as reações das mães (Jaramillo et al., 2017). Nesse sentido, estudos sobre essa temática devem ser realizados em diferentes contextos culturais. 0 único estudo que investigou relações semelhantes no Brasil foi conduzido por Lins (2018), que constatou correlações positivas entre os quatro tipos de reações não apoiadoras e os problemas internalizantes. Embora o estudo não tenha avaliado diretamente a autorregulação emocional infantil, os problemas internalizantes incluem dificuldades emocionais como a ansiedade, a depressão e a reatividade emocional, portanto, seus achados fornecem apoio indireto ao impacto desfavorável das reações não apoiadoras.
Paula Kleize Costa Sales e Patrícia Alvarenga

Em resumo, a literatura mostra evidências sobre as relações entre reações parentais apoiadoras e não apoiadoras da expressividade emocional dos filhos e a capacidade de autorregulação emocional infantil (Breaux et al., 2017; Meyer et al., 2014; Miller-Slough et al., 2016; Mirabile, 2015; Morelen \& Suveg, 2012; Premo \& Kiel, 2014; Shewark \& Blandon, 2015), porém, achados controversos (Han et al., 2015; McCord \& Raval, 2016; Rogers et al., 2016), indicam a potencial influência de diferenças socioculturais (Hooper et al., 2018; Jaramillo et al., 2017; Morelen \& Thomassin, 2013). Ademais, os estudos raramente avaliam estratégias de autorregulação emocional relatadas pelas próprias crianças (Morelen et al., 2014) assim como discutem pouco os possíveis efeitos da autorregulação emocional infantil sobre as reações maternas (Premo \& Kiel, 2014). Por fim, pouco se sabe acerca da relação entre as reações maternas às emoções negativas dos filhos e a autorregulação em amostras de famílias brasileiras (Lins, 2018). Assim, o objetivo deste estudo foi investigar as relações entre as reações maternas às emoções negativas dos filhos e a autorregulação emocional da criança. A autorregulação emocional da criança foi avaliada tanto pelo relato materno como pelo relato das próprias crianças. Foram testadas duas hipóteses: 1) as reações maternas apoiadoras estão positivamente correlacionadas ao escore de autorregulação emocional infantil relatado pela mãe e à frequência de estratégias de enfrentamento relatadas pela criança e 2) as reações não apoiadoras estão negativamente correlacionadas ao escore de autorregulação emocional infantil relatado pela mãe e positivamente correlacionadas à frequência de estratégias de fuga relatadas pela criança.

\section{MÉTODO}

\section{PARTICIPANTES}

Trinta e três mães e 30 crianças participaram deste estudo. A amostra foi selecionada para um estudo mais amplo que avaliou os efeitos de uma intervenção para promover as práticas maternas de socialização emocional e o desenvolvimento emocional da criança. 0 critério de inclusão das mães no presente estudo foi residir com a criança no mesmo domicílio e o critério de exclusão foi a presença de transtornos mentais graves nas mães e de doenças crônicas e de síndromes genéticas nos filhos. 0 critério de inclusão das crianças foi a participação da mãe no estudo, sendo que três foram excluídas por terem sido entrevistadas após o início da intervenção com suas mães.

Quanto aos dados sociodemográficos das mães, a cor/ raça que as mães participantes do estudo mais declararam pertencer foi a preta, 60,6\%, seguida pela parda, 27,3\%, branca, $9,1 \%$, e indígena, $3 \%$. As mães tinham, em média, 35,15 anos 


\section{H* INTERACÃO EM PSICOLOGIA}

de idade $(D P=5,19)$ e 12,64 anos de escolaridade $(D P=2,31)$. Cerca de sessenta e três por cento das mães exerciam alguma atividade remunerada, 30,3\% não exerciam atividade remunerada e duas mães estavam afastadas do trabalho devido a problemas de saúde. Do total de mães participantes, $36,4 \%$ possuíam algum problema de saúde.

Em relação aos dados sobre os filhos das mães participantes, 23 são meninas $(69,7 \%)$ e 10 são meninos (30,3\%). De acordo com os relatos das mães, a maioria das crianças tinha a cor/raça preta, 36,4\%, e parda, 36,4\%. Aproximadamente, dezoito por cento era branca, $6,1 \%$ era amarela e $3 \%$ era indígena. A média de idade dos filhos (as) em meses foi equivalente a 83,03 $(D P=6,72)$. Quanto à escolaridade desses filhos, $54,5 \%$ cursavam o primeiro ano do ensino fundamental e $45,5 \%$ cursavam o segundo ano. No que concerne à existência de irmãos, $60,6 \%$ das crianças possuíam ao menos um irmão e $39,4 \%$ das crianças não tinham nenhum irmão. Sobre o principal cuidador da criança, para $69,7 \%$ dos casos, a própria mãe era a principal cuidadora. Para outros $15,2 \%$ dos casos, a avó materna era a principal cuidadora e para 6,1\% das crianças, o pai era o principal cuidador. Para os outros $9 \%$ restantes, os principais cuidadores da criança eram a avó paterna (3\%), a babá (3\%) e uma amiga da família (3\%). Em relação à existência de problemas de saúde entre as crianças, somente $3 \%$ possuía algum problema. Entre as 30 crianças que responderam à entrevista, do total de 33 que representam os filhos das mães participantes, 20 são meninas (66,7\%) e 10 são meninos (33,3\%).

Quanto ao genitor da criança, dois pais haviam falecido antes do momento da coleta de dados. No que se refere aos outros 31 pais, a idade média foi de 39,94 anos ( $D P=7,92$ ). Os pais estudaram, em média, durante 11,47 anos $(D P=3,81)$. Destaca-se que uma mãe não soube informar a escolaridade do pai da criança. Aproximadamente, sessenta por cento dos pais residiam com a mãe e a criança, 33,3\% não moravam com a mãe da criança. Cerca de $75,8 \%$ dos pais exerciam alguma atividade remunerada e $18,2 \%$ não exerciam qualquer atividade financeira.

Por fim, no que se refere às características das famílias, a renda familiar média foi de $\mathrm{R} \$ 3.412,63(D P=2.284,68)$ e as residências familiares abrigavam, em média, quatro moradores. Mais detalhes acerca das características sociodemográficas da amostra podem ser consultados na Tabela 1.

\section{INSTRUMENTOS}

Ficha de Dados Sociodemográficos. A ficha foi utilizada para coletar dados que caracterizam a amostra, tais como idade, nível de escolaridade, renda, sexo das crianças, quantidade de filhos e outras informações.

\section{AVALIACCÃO DAS REAÇÕES MATERNAS ÀS EMOÇÕES NEGATIVAS DOS FILHOS}

Escala de Reações Parentais às Emoções Negativas dos FiIhos (CCNES). A CCNES é uma escala desenvolvida por Fabes et al. (1990) e adaptada por Lins et al. (2017) para uso no Brasil. A CCNES apresenta doze situações hipotéticas cotidianas nas quais a criança pré-escolar e escolar exprime emoções negativas diante de eventos frustrantes. A versão brasileira da CCNES contém duas escalas: reações apoiadoras e reações não apoiadoras (Lins et al., 2017). Essas escalas são divididas em sete subescalas. A escala de reações apoiadoras contém as subescalas: a) reações centradas no problema, b) reações incentivadoras e c) reações centradas na emoção. A escala de reações não apoiadoras inclui as subescalas: d) reações de desconforto, e) reações punitivas, f) reações minimizadoras e g) reações de indiferença. 0 escore de cada subescala é obtido a partir do cálculo da média da pontuação dos itens correspondentes (Lins et al., 2017). De acordo com Lins et al. (2017), os valores de alpha da CCNES obtidos no estudo brasileiro foram adequados e próximos àqueles atingidos no estudo de Fabes et al. (2002), com poucas exceções. Após a remoção dos quatro itens negativos da subescala reações de desconforto, realizada por Lins et al. (2017), o alpha dessa subescala aumentou para 0,46 . Com base nesse achado, optou-se por calcular o escore da subescala de reações de desconforto no presente estudo sem incluir as respostas aos itens negativos. 0 alpha da subescala das reações centradas no problema foi equivalente a 0,60 . As reações centradas na emoção e as reações incentivadoras tiveram alpha igual a 0,74 . As reações punitivas, as reações minimizadoras e as reações de indiferença obtiveram os seguintes valores de alpha, respectivamente: $0,73,0,77$ e 0,69.

\section{AVALIAÇÃO DA AUTORREGULAÇÃO EMOCIONAL INFANTIL}

Inventário de Autorregulação Emocional (ERC). O Inventário de Autorregulação Emocional foi proposto por Shields \& Cicchetti (1995). O Inventário de Autorregulação Emocional contém 24 itens que podem ser respondidos por um adulto próximo à criança. Os itens da ERC compõem duas subescalas, a regulação emocional e a labilidade/negatividade emocional. A subescala da regulação emocional avalia a consciência das próprias emoções e a expressividade emocional positiva (por exemplo, "não permanece quieta ou mal-humorada, ansiosa ou triste após eventos emocionalmente estressantes") (Reis et al., 2016). A subescala labilidade emocional avalia o nível de ativação emocional, de desregulação da raiva e de reatividade, a ausência de flexibilidade e a labilidade do humor (por exemplo, "É propensa a explosões de raiva/birra") (Reis et al., 2016). No presente estudo, só foi considerado o escore total da autorregulação emocional. A pontuação mínima no ERC é 
Tabela 1. Características Sociodemográficas dos Participantes $(N=33)$

\begin{tabular}{|c|c|c|c|}
\hline Característica & Mãe (n = 33) & Criança $(n=33)$ & Pai $(n=31)^{b}$ \\
\hline \multicolumn{4}{|l|}{ Cor/Raça } \\
\hline Preta & $60,6 \%(20)$ & $36,4 \%(12)$ & \\
\hline Parda & $27,3 \%(9)$ & $36,4 \%(12)$ & \\
\hline Branca & $9,1 \%(3)$ & $18,2 \%(6)$ & \\
\hline Indígena & $3 \%(1)$ & $3 \%(1)$ & \\
\hline Amarela & & $6,1 \%(2)$ & \\
\hline Idade ${ }^{a}$ & $M=35,15(D P=5,19)$ & $M=83,03(D P=6,72)$ & $M=39,94(\mathrm{DP}=7,92)$ \\
\hline \multicolumn{4}{|l|}{ Sexo } \\
\hline Feminino & & $69,7 \%(23)$ & \\
\hline Masculino & & $30,3 \%(10)$ & \\
\hline Escolaridade (anos) & $M=12,64(D P=2,31)$ & & $M=11,47(D P=3,81)$ \\
\hline \multicolumn{4}{|l|}{ Série escolar } \\
\hline $1^{\circ}$ ano & & $54,5 \%(18)$ & \\
\hline $2^{\circ}$ ano & & $45,5 \%(15)$ & \\
\hline \multicolumn{4}{|l|}{ Atividade remunerada } \\
\hline Sim & $63,6 \%(21)$ & & $75,8 \%(25)$ \\
\hline Não & $30,3 \%(10)$ & & $18,2 \%(6)$ \\
\hline Afastado(a) pelo INSS & $6,1 \%(2)$ & & \\
\hline \multicolumn{4}{|l|}{ Problema de saúde } \\
\hline Sim & $36,4 \%(12)$ & $3 \%(1)$ & \\
\hline Não & $63,6 \%(21)$ & $97 \%(32)$ & \\
\hline \multicolumn{4}{|l|}{ Coabitação parental } \\
\hline Sim & $60,6 \%(20)$ & & \\
\hline Não & $33,3 \%(11)$ & & \\
\hline Número de moradores & $M=4,61(D P=1,29)$ & & \\
\hline Renda familiar (reais) & $M=3.412,63(D P=2.284,68)$ & & \\
\hline \multicolumn{4}{|l|}{ Irmãos } \\
\hline Sim & & $60,6 \%(20)$ & \\
\hline Não & & $39,4 \%(13)$ & \\
\hline \multicolumn{4}{|l|}{ Principal cuidador(a) } \\
\hline Mãe & & $69,7 \%(23)$ & \\
\hline Pai & & $6,1 \%(2)$ & \\
\hline Avó Materna & & $15,2 \%(5)$ & \\
\hline Avó Paterna & & $3 \%(1)$ & \\
\hline Babá & & $3 \%(1)$ & \\
\hline Amiga da família & & $3 \%(1)$ & \\
\hline
\end{tabular}

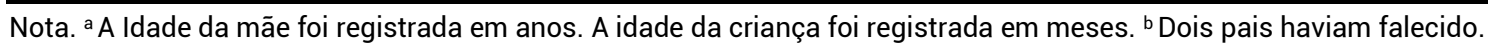

igual a 23 e o escore máximo possível é igual a 92. Reis et al. (2016) verificaram que a consistência interna da versão brasileira da escala, com 23 itens, também é apropriada, $a=0,86$ Este instrumento será referido como relato materno da autorregulação emocional infantil.

Entrevista para Avaliação das Estratégias de Autorregulação Emocional Infantil. A entrevista foi elaborada pelas autoras deste estudo com base nas propostas de Dias et al. (2000) e Cruvinel e Boruchovitch (2004, 2010, 2011). A entrevista consistiu na exibição de três trechos do filme infantil "Ozzy", no qual um cachorro expressa emoções negativas (tristeza, raiva e medo). Após a exibição de cada uma das cenas, o pesquisador exibia o print da tela em que o personagem exibia a expressão facial da emoção investigada e fazia as seguintes perguntas: "O que o cachorro está sentindo? É bom ou é ruim sentir isso? Você já sentiu (nome da emoção usado pela 


\section{M." INTERACÃO EM PSICOLOGIA}

criança)?"Depois, as estratégias de autorregulação das três emoções foram investigadas pelas seguintes questões: "Você lembra quando foi a última vez que você sentiu (nome da emoção usado pela criança)? 0 que você fez? O que você faz quando você sente (nome da emoção usado pela criança)?".

As respostas das crianças à entrevista foram analisadas por meio da análise de conteúdo quantitativa (Bardin, 1977; Laville \& Dionne, 1999). A estrutura de categorias foi desenvolvida com base nos estudos de Meyer et al. (2014), Cruvinel e Boruchovitch $(2010,2011)$ e outros autores (Aldao et al., 2010; Dunsmore et al., 2012; Zeman et al., 2006). A estrutura classifica as estratégias de autorregulação em dois agrupamentos: estratégias de enfrentamento e estratégias de fuga. As estratégias de enfrentamento caracterizam-se por vivenciar, tolerar, validar, compreender as emoções e obter mais informações sobre elas, sem abandonar as situações que provocam a experiência emocional negativa. Três categorias compuseram esse agrupamento: a) controle do comportamento e da emoção (visam dominar a tendência de ação relacionada à vivência emocional. Ex.: "Fiquei com muita raiva, eu quase bati nele, mas eu não bati porque ele ia bater"); b) busca de apoio afetivo-social (tentativas da criança de manter proximidade e interagir com a intenção de obter ajuda para lidar com as emoções. Ex.: "Eu falei com a diretora") e c) resolução de problema (resolução do conflito gerador da emoção. Ex.: "É. Foi porque, quando foi embora aí eu fiquei com muita saudade dele. Aí eu disse "pô, por que você não foi lá em casa?"). As estratégias de fuga caracterizam-se por tentativas de conter ou eliminar reações corporais relacionadas às emoções negativas, evitar a situação que as elicita, tentar vivenciar emoções positivas ou prazerosas, ou ainda agredir ao experimentar uma emoção negativa. Este agrupamento foi formado por quatro categorias: a) distração/afastamento (busca por atividades prazerosas ou distrações que retirem a criança fisicamente da situação geradora da emoção. Ex.: "Entrei na minha casa e fui assistir pra tirar isso"); b) alteração do pensamento (modificar o conteúdo do pensamento associado à emoção negativa. Ex.: "Fico pensando que a boneca é minha mãe"); c) agressividade (comportamentos agressivos direcionados a si própria, a algum objeto ou a outras pessoas. Ex.: "Taquei areia nele de volta") e d) Supressão emocional (impedem, escondem ou disfarçam reações corporais típicas de emoções negativa tais como chorar ou ruborizar. Ex.: "Escondi meu choro").

Duas codificadoras independentes que desconheciam o objetivo e as hipóteses do estudo receberam treinamento (30 horas) e realizaram a análise de conteúdo. 0 índice de fidedignidade entre observadores foi 0,86 (Kappa de Cohen), que é considerado excelente (Robson, 1993).

\section{PROCEDIMENTOS}

Coleta de dados. Após a aprovação pelo Comitê de Ética em Pesquisa [informação suprimida para não identificar o(s) autor(es)], mães cujos filhos estavam matriculados em duas escolas da rede particular de ensino da [informação suprimida para não identificar o(s) autor(es)], com mensalidades até R\$ 545,00 foram convidadas para participar do estudo. A coleta dos dados foi realizada com cada mãe em uma sala reservada na própria escola e durou aproximadamente 45 minutos. As mães assinaram o Termo de Consentimento Livre e Esclarecido - TCLE e responderam à Ficha de Dados Sociodemográficos, à Escala de Reações Parentais às Emoções Negativas dos Filhos - CCNES e ao Inventário de Autorregulação Emocional - ERC. As mães responderam aos instrumentos individualmente, em forma de entrevista. 0 entrevistador lia os itens e as mães os respondiam com o auxílio de cartões de resposta. Após completar a coleta de dados com as mães, os filhos das participantes foram convidados para participar também do estudo. As crianças que concordaram e assentiram verbalmente responderam, em seguida, a Entrevista para Avaliação das Estratégias de Autorregulação Emocional Infantil. Todas as entrevistas foram gravadas em áudio e transcritas para análise. Este estudo envolveu risco mínimo tanto para as mães quanto para as crianças participantes, relacionado à evocação de memórias e de emoções difíceis. Nos casos em que a mãe apresentou desconforto psicológico, a coleta foi interrompida e em seguida foram usadas estratégias para acolher e confortá-la. Além disso, participantes foram encaminhados para Serviços de Psicologia ou foi oferecida a possibilidade de atendimento psicológico por meio de um projeto de extensão universitária coordenado pela segunda autora deste estudo.

Análise dos dados. Inicialmente foram realizadas análises descritivas, incluindo média, desvio padrão e intervalo de confiança (95\%) dos escores gerados pelas CCNES e ERC e das estratégias de autorregulação emocional relatadas pela criança durante a entrevista. Em seguida, foram utilizados testes de correlação bivariada. 0 teste de Pearson foi adotado com os dados gerados pela CCNES e ERC devido à distribuição simétrica verificada nas variáveis. 0 teste de Spearman foi utilizado com os dados gerados pela entrevista em função da sua distribuição assimétrica. 0 ponto de corte utilizado para a avaliação da significância de todos os valores de alpha foi 0,05 .

\section{RESULTADOS}

Resultados das análises de estatística descritiva indicaram que as reações maternas às emoções negativas dos filhos foram predominantemente apoiadoras. A média dessas 
reações foi igual a 4,02 $(D P=0,31 ; I C[3,90-4,13])$. A média das reações não apoiadoras, por sua vez, foi equivalente a $2,08(D P=0,53$; IC $[1,88-2,27])$. Entre os sete tipos de reações analisadas, as reações centradas na emoção obtiveram a maior média, 4,43 ( $D P=0,38$; IC [4,30 - 4,57]). Em contrapartida, a menor média foi verificada nas reações de indiferença, que foi de $1,47(D P=0,36 ;$ IC $[1,34-1,60])$. No que se refere às outras reações apoiadoras, a média das reações centradas no problema foi de 4,40 ( $D P=0,33$; IC [4,29 - 4,52]) e a das reações incentivadoras foi de 3,22 ( $D P=0,44 ;$ IC [3,07 - 3,38]). Em relação às outras subescalas das reações não apoiadoras, a média das reações punitivas foi igual a $2,45(D P=0,77$; IC [2,17 - 2,72]), a das reações de desconforto foi equivalente a $2,25(D P=0,82 ;$ IC $[1,95-2,54])$ e as reações minimizadoras obtiveram média igual a 2,28 $(D P=0,85 ; I C[1,97-2,58])$. A média do escore geral do relato materno de autorregulação emocional infantil foi equivalente a $72,00(D P=6,67 ; / C[69,63$ - 74,36]). Quanto aos dados da entrevista respondida pelas crianças, a média das estratégias de enfrentamento foi igual a $1,65(D P=1,42 ; I C[1,11-2,19])$ e das estratégias de fuga foi equivalente a $2,31(D P=1,77 ; I C[1,63-2,98])$.
Resultados do teste de correlação de Pearson revelaram as seguintes correlações entre o escore total de autorregulação emocional infantil relatado pela mãe e as reações não apoiadoras: reações punitivas $(r=-0,33 ; p<0,05)$, reações de desconforto $(r=-0,32 ; p<0,05)$, reações de indiferença $(r=$ $-0,37 ; p<0,05)$, reações minimizadoras $(r=-0,31 ; p<0,05) \mathrm{e}$ total de reações não apoiadoras $(r=-0,43 ; p<0,01)$. Por outro lado, resultados das análises de correlação de Spearman entre as reações e o relato das crianças das estratégias de autorregulação emocional revelaram que o agrupamento das estratégias de enfrentamento teve correlação negativa com as reações de desconforto $(\rho=-0,31 ; p<0,05)$ e correlação positiva com as reações incentivadoras $(\rho=0,36 ; p<0,05)$. Por último, o agrupamento das estratégias de fuga esteve negativamente correlacionado com as reações incentivadoras ( $\rho=$ $-0,34 ; p<0,05)$ e positivamente correlacionado com as reações de desconforto $(\rho=0,31 ; p<0,05)$ e com o agrupamento das reações não apoiadoras $(\rho=0,32 ; p<0,05)$. A Tabela 2 apresenta os resultados dessas análises de correlação. As demais relações entre as reações maternas às emoções negativas dos filhos e a autorregulação emocional infantil não foram significativas.

Tabela 2. Correlações entre as Reações Maternas às Emoções Negativas dos Filhos e a Autorregulação Emocional Infantil

\begin{tabular}{|c|c|c|c|c|c|c|c|c|c|c|c|c|}
\hline Variáveis & 1 & 2 & 3 & 4 & 5 & 6 & 7 & 8 & 9 & 10 & 11 & 12 \\
\hline \multicolumn{13}{|l|}{ Reações maternas } \\
\hline 1. Punitivas & _- & & & & & & & & & & & \\
\hline 2. Desconforto & $0,59 * *$ & - & & & & & & & & & & \\
\hline 3. Indiferença & $0,37 *$ & $0,51 * *$ & - & & & & & & & & & \\
\hline 4. Minimizadoras & $0,76 * *$ & $0,77 * *$ & $0,50 * *$ & _- & & & & & & & & \\
\hline $\begin{array}{l}\text { 5. Centradas no } \\
\text { problema }\end{array}$ & 0,10 & $-0,24$ & $-0,27$ & $-0,20$ & - & & & & & & & \\
\hline $\begin{array}{l}\text { 6. Centradas na } \\
\text { emoção }\end{array}$ & 0,26 & 0,05 & 0,23 & 0,12 & $0,47 * *$ & - & & & & & & \\
\hline 7. Incentivadoras & $-0,15$ & $-0,31$ & $-0,17$ & $-0,23$ & $0,48 * *$ & 0,34 & - & & & & & \\
\hline 8. Total apoiadoras & 0,08 & $-0,22$ & $-0,07$ & $-0,12$ & $0,79 * *$ & $0,74 * *$ & $0,80 * *$ & - & & & & \\
\hline $\begin{array}{l}\text { 9. Total não apoiadoras } \\
\text { Autorregulação } \\
\text { infantil }\end{array}$ & $0,84 * *$ & $0,83^{* *}$ & $0,63^{* *}$ & $0,93 * *$ & $-0,19$ & 0,20 & $-0,27$ & $-0,11$ & - & & & \\
\hline 10. Escore (relato mãe) & $-0,33 *$ & $-0,32 *$ & $-0,37 *$ & $-0,31 *$ & $-0,17$ & $-0,13$ & 0,06 & $-0,09$ & $-0,43 * *$ & - & & \\
\hline $\begin{array}{l}\text { 11. Enfrentamento } \\
\text { (relato criança) }^{a}\end{array}$ & $-0,04$ & $-0,31 *$ & $-0,28$ & $-0,29$ & 0,16 & 0,10 & $0,36 *$ & 0,26 & $-0,27$ & $0,53 * *$ & - & \\
\hline $\begin{array}{l}\text { 12. Fuga (relato } \\
\text { criança) }^{a}\end{array}$ & 0,06 & 0,31 * & 0,29 & 0,27 & $-0,17$ & $-0,21$ & $-0,34 *$ & $-0,25$ & $0,32 *$ & $-0,29$ & $-0,42 *$ & - \\
\hline
\end{tabular}

Nota. $N=33$.

${ }^{a} N=30$ Correlação de Spearman.

$* p<0.05 * * p<0.01$ 


\section{H* INTERACÃO EM L* PSICOLOGIA}

\section{DISCUSSÃO}

Este estudo evidenciou relações entre as reações apoiadoras e não apoiadoras das mães à expressão emocional dos filhos e a autorregulação emocional infantil em uma amostra brasileira, considerando tanto o relato da mãe quanto o da criança ao examinar a autorregulação. Os achados forneceram maior apoio à noção de que as reações não apoiadoras se relacionam a menor capacidade de autorregulação emocional infantil.

A hipótese de que as reações maternas apoiadoras às emoções negativas dos filhos estão positivamente correlacionadas ao relato materno do escore de autorregulação emocional infantil e ao relato de estratégias de enfrentamento por parte da criança recebeu pouco apoio dos resultados, indicando que somente as reações incentivadoras estiveram correlacionadas à autorregulação relatada pelas crianças. Contudo, não houve correlações significativas entre as reações maternas apoiadoras às emoções dos filhos e o relato materno do escore de autorregulação emocional da criança.

É possível que mães que respondem às expressões de emoções negativas encorajando ou validando a expressão e a experiência de emoções negativas ensinem a criança a enfrentar a situação buscando apoio para compreender e lidar com essas experiências emocionais. Esse tipo de manejo pode levar a criança a expressar emoções de modo socialmente aceitável, sofrer menos com a vivência de emoções negativas e aprender mais sobre a experiência emocional. Sabatier et al. (2017) afırmam que, ao fazer uso da estratégia busca de apoio, crianças que possuem cerca de sete anos de idade são mais capazes de controlar as emoções por meio de estratégias verbais em vez de expressá-las por meio de comportamentos indesejáveis. Além disso, as trocas interpessoais podem colaborar para a redução de sensações desagradáveis (Whitebread \& Basilio, 2012). Com as interações favorecidas a partir do uso da estratégia de busca de apoio, a criança também pode obter diversas explicações sobre as situações nas quais determinada emoção tende a ocorrer. Essas informações parecem desempenhar um papel importante na compreensão e na expressão emocional (Souza \& Mendes, 2018). Sob outra perspectiva, crianças que usam mais estratégias de enfrentamento podem evocar nas mães mais reações apoiadoras. As mães podem, então, passar a escutar mais o relato da criança quando vivencia emoções negativas e fornecer ajuda nessas situações. Por meio do uso das estratégias de enfrentamento, assim, a criança pode comunicar à mãe as suas dificuldades e necessidades e mobilizá-la a transmitir conhecimento acerca das experiências emocionais e de como lidar com elas (Miller-Slough et al., 2016; Premo \& Kiel, 2014).
Quanto à ausência de relações entre as reações apoiadoras e o relato materno do escore de autorregulação emocional, esse resultado assemelha-se àquele encontrado por Lins (2018), que avaliou a autorregulação emocional indiretamente com uma amostra brasileira de díades com características sociodemográficas semelhantes. A autora não verificou correlações entre as reações maternas apoiadoras e os problemas internalizantes, que se referem a comportamentos que podem indicar dificuldades relacionadas à autorregulação das emoções. No entanto, tanto os resultados do presente estudo quanto aqueles encontrados por Lins (2018) não são compatíveis com os achados do estudo de Han et al. (2015), conduzido na China. Essa diferença pode ser explicada por fatores socioculturais (Jaramillo et al., 2017; Lins \& Alvarenga, 2018). Assim, as mães do presente estudo podem ter respondido a escala de reações apoiadoras com base em crenças acerca de como seria a maneira de reagir às expressões de emoções negativas dos filhos mais passível de aprovação e validação social e não de acordo com o que ocorre, efetivamente.

A segunda hipótese testada, de que as reações não apoiadoras estão negativamente correlacionadas ao relato materno do escore de autorregulação emocional infantil e positivamente correlacionadas ao relato de estratégias de fuga por parte da criança foi corroborada. De acordo com os resultados das análises de correlação, todas as reações não apoiadoras estiveram negativamente correlacionadas com o relato materno do escore de autorregulação emocional infantil. Além disso, as reações de desconforto e o agrupamento das reações não apoiadoras tiveram correlação positiva com o relato da criança de estratégias de fuga. Achados semeIhantes foram obtidos em investigações realizadas em outros países (Miller-Slough et al., 2016; Shewark \& Blandon, 2015) e no estudo de Lins (2018), realizado em contexto brasileiro. É provável que as reações não apoiadoras possam ser compreendidas como tentativas de evitação de contato com as experiências emocionais negativas nos filhos, devido à valorização da vivência de emoções positivas e à desvalorização do sofrimento e da experiência de emoções negativas (Carrera, 2014; Mendes \& Oliveira, 2013). No entanto, o uso das reações não apoiadoras pode ensinar estratégias de autorregulação que geram prejuízos ou inibem o desenvolvimento emocional infantil. Assim, as crianças cujas mães utilizam reações não apoiadoras podem suprimir a expressão emocional (Eisenberg et al., 1998; Fabes et al., 2002; Lins \& Alvarenga, 2018), fazer uso de estratégias que evitam ou eliminam a vivência de emoções negativas ou expressar as emoções negativas de modo cada vez mais exacerbado (Lins \& Alvarenga, 2018). Em longo prazo, o uso contínuo dessas estratégias pode produzir consequências problemáticas como, por exemplo, o aumento da intensidade da experiência emocional (Hayes et al., 1999). É possível também que o uso das reações não apoiadoras 


\section{MLE INTERACÃO EM PSICOLOGIA}

torne mais provável o uso de comportamentos agressivos como forma de lidar com emoções negativas. A partir de um estudo realizado com mães afro-americanas, Hooper et al. (2018) verificaram que as reações de desconforto foram associadas a maiores escores de problemas externalizantes. Em contrapartida, também é provável que crianças que têm mais dificuldade de regular as emoções ou que expressam emoções negativas com alta frequência façam com que as mães tenham mais reações não apoiadoras. Mães cujos filhos (as) têm reações emocionais exacerbadas frequentemente podem se tornar menos sensíveis às expressões emocionais das crianças. Essas mães podem ser também mais propensas a ignorar ou a reagir com outros tipos de reações não apoiadoras às expressões de emoções negativas pelas crianças como forma de controlar tais expressões e de reduzir o incômodo que a expressão dessas emoções pode causar. Assim, é esperado que, com o tempo, tanto as reações maternas não apoiadoras quanto a expressão emocional não adequada nas crianças sejam mais frequentes (Breaux et al.,2017; Lins \& Alvarenga, 2018; Morelen \& Suveg, 2012).

Aspectos metodológicos relevantes deste estudo merecem ser destacados. Dados acerca da autorregulação das emoções das crianças foram obtidos por meio de duas fontes distintas, relatos da mãe e da criança. Este estudo também constitui um dos raros esforços para a obtenção de dados acerca da autorregulação emocional infantil a partir da percepção da criança. De qualquer modo, este estudo também apresenta limitações, que se referem à pequena quantidade de participantes, à impossibilidade de realizar inferências causais em vista do tipo de delineamento adotado e às baixas frequências de estratégias relatadas pelas crianças, que inviabilizaram a análise das categorias mais específicas. Nesse sentido, sugere-se que as relações entre as reações maternas às emoções negativas dos filhos e a autorregulação emocional infantil continuem sendo exploradas no Brasil, especialmente devido às evidências do impacto de fatores culturais sobre essa problemática (Mendes \& Oliveira, 2013).

Em resumo, este estudo gerou novas evidências acerca das relações entre as reações maternas às emoções dos filhos e a autorregulação emocional infantil, com relevantes contribuições para a Psicologia do desenvolvimento e sua interface com a Psicologia clínica. Em conjunto, os achados indicam que as reações das mães, que constituem um meio de heterorregulação da expressão emocional infantil, estimulam na criança o uso de meios de autorregulação emocional semelhantes. Isto é, crianças que convivem com pais menos habilidosos para lidar com as emoções tendem a adotar estratégias de fuga e a revelar menor capacidade de enfrentamento de emoções perturbadoras, enquanto crianças que experimentam reações apoiadoras com maior frequência desenvolvem maior capacidade de lidar com essas vivências. Quanto às implicações práticas, esses achados podem se traduzir em estratégias de intervenção e beneficiar famílias. Assim, recomenda-se a realização de ações psicoeducativas com propósito de divulgar e discutir informações acerca de possíveis impactos das reações apoiadoras e não apoiadoras sobre a autorregulação emocional da criança em distintos veículos de comunicação e serviços, sobretudo naqueles voltados para a preparação ou o desenvolvimento de competências parentais.

\section{AGRADECIMENTOS}

Os autores agradecem a Sara Correia e a Milena MagaIhães pelo apoio na análise dos dados da entrevista.

\section{CONTRIBUIÇÃO DE CADA AUTOR}

Certificamos que todos os autores participaram suficientemente do trabalho para tornar pública sua responsabilidade pelo conteúdo. A contribuição de cada autor pode ser atribuída como se segue:

$P$.K.C.S. e $P$.A. são responsáveis pela conceitualização, investigação, redação e visualização do artigo; P.K.C.S realizou a tabulação e a análise dos dados; P.A. é responsável pela administração do projeto, supervisão e revisão e edição.

\section{DECLARAÇÃO DE CONFLITOS DE INTERESSES}

Os autores declaram que não há conflitos de interesse no manuscrito submetido [do Instituto de Psicologia da Universidade Federal da Bahia (parecer no 3.526.867)] [da cidade de Salvador].

\section{DECLARAÇÃO DE FINANCIAMENTO}

A pesquisa relatada no manuscrito foi financiada parcialmente pela bolsa de mestrado da primeira autora (CAPES, prot. 88882.183178/2018-01) e pelo apoio financeiro da segunda autora recebido pelos editais CHAMADA UNIVERSAL MCTI/ CNPq No 01/2016 (processo 400395/2016-9) e CNPq/Produtividade em Pesquisa - PQ 2015 (processo 307263/2015-0).

\section{REFERÊNCIAS}

Aldao, A., Nolen-hoeksema, S., \& Schweizer, S. (2010). Emotion-regulation strategies across psychopathology: a meta-analytic review. Clinical Psychology Review, 30(2), 217237. https://doi.org/10.1016/j.cpr.2009.11.004

Bardin, L. (1977). Análise de conteúdo. Edições 70. 


\section{H MTERAC̄OOEM ET PSICOLOGIA}

Breaux, R. P., Mcquade, J. D., Harvey, E. A., \& Zakarian, R. J. (2017). Longitudinal associations of parental emotion socialization and children's emotion regulation: the moderating role of ADHD symptomatology. Journal of Abnormal Child Psychology, 46(4), 671-683. https://doi.org/10.1007/ s10802-017-0327-0

Bridges, L. J., Denham, S. A., \& Ganiban, J. M. (2004). Definitional issues in emotion regulation research. Child Development, 75(2), 340-345. https://doi.org/10.1111 /j.1467-8624.2004.0067

Carrera, F. (2014). O imperativo da felicidade em sites de redes sociais: materialidade como subsídio para o gerenciamento de impressões (quase) sempre positivas. Eptic Online, 16(1), 33-44. https://seer.ufs.br/index.php/eptic/ article/view/1857

Carthy, T., Horesh, N., Apter, A., Edge, M. D., \& Gross, J. J. (2010). Emotional reactivity and cognitive regulation in anxious children. Behaviour Research and Therapy, 48, 384393. https://doi.org/10.1016/j.brat.2009.12.013.

Cruvinel, M., \& Boruchovitch, E. (2004). Entrevista e pranchas para avaliação da regulação emocional de alunos do ensino Fundamental - ERE. Disponibilizado pelas autoras.

Cruvinel, M., \& Boruchovitch, E. (2010). Regulação emocional: a construção de um instrumento e resultados iniciais. Psicologia em Estudo, 15(3), 537-545. https://doi. org/10.1590/s1413-73722010000300011

Cruvinel, M., \& Boruchovitch, E. (2011). Regulação emocional em crianças com e sem sintomas de depressão. Estudos de Psicologia, 16(3), 219-226. https://doi.org/10.1590/ s1413-294x2011000300003

Dias, M. G. B. B., Vikan, A., \& Gravas, S. (2000). Tentativa de crianças em lidar com as emoções de raiva e tristeza. Estudos de Psicologia, 5(1), 49-70. https://doi.org/10.1590/ s1413-294x2000000100004

Dunsmore, J. C., Booker, J. A., \& Ollendick, T. H. (2012). Parental emotion coaching and child emotion regulation as protective factors for children with oppositional defiant disorder. Social Development, 22(3), 1-23. https://doi.or$\mathrm{g} / 10.1111 / \mathrm{j} .1467-9507.2011 .00652 . x$

Eisenberg, N., Cumberland, A., \& Spinrad, T. L. (1998). Parental socialization of emotion. Psychological Inquiry, 9(4), 241273. https://doi.org/10.1207/s15327965pli0904_1

Fabes, R. A., Eisenberg, N., \& Bernzweig, J. (1990). The coping with children's negative emotion scale: procedures and scoring. Arizona State University. https://doi.org/10.1037/ t36591-000

Fabes, R. A., Poulin, R. E., Eisenberg, N., \& Madden-Derdich, D. A. (2002). The coping with children's negative emotions scale (CCNES): Psychometric properties and relations with children's emotional competence. Marriage \& Family Review, 34(3-4), 285-310. https://doi.org/10.1300/ j002v34n03_05
Frijda, N. H. (2008). The Psychologists' Point of View. In M. Lewis, J. M. Haviland-jones, \& Barrett, (Eds.), Handbook of Emotions ( $3^{\mathrm{a}}$ ed.) (68-87). The Guilford Press.

Han, Z. R. Qian, J., Gao, M., \& Dong, J. (2015). Emotion socialization mechanisms linking chinese fathers', mothers', and children's emotion regulation: a moderated mediation. Journal of Child and Family Studies, 24, 3570-3579. https:// doi.org/10.1007/s10826-015-0158-y.

Hayes, S. C., Strosahl, K., \& Wilson, K. G. (1999). Acceptance and commitment therapy: an experimental approach to behavior change. Guilford.

Hooper, E. G., Wu, Q., Ku, S., Gerhardt, M., \& Feng, X. (2018). Maternal emotion socialization and child outcomes among african americans and european Americans. Journal of Child and Family Studies, 27(6), 1870-1880. https:// doi.org/10.1007/s10826-018-1020-9

Jaramillo, J. M., Rendón, M. I., Muñoz, L., Weis, M., \& Trommsdorff, G. (2017). Children's self-regulation in cultural contexts: the role of parental socialization theories, goals, and practices. Frontiers in Psychology, 8. https://doi. org/10.3389/fpsyg.2017.00923

Laville, C., \& Dionne, J. (1999). A construção do saber: manual de metodologia da pesquisa em ciências humanas (L. M. Siman, Trad.). Artes Médicas.

Lins, T. C. S., Alvarenga, P., Mendes, D. M. L. F., \& Pessôa, L. F. (2017). Adaptação da Coping with Children's Negative Emotions Scale (CCNES). Avaliação Psicológica, 16(2), 196-204. https://doi.org/10.15689/AP.2017.1602.10

Lins, T. C. S., \& Alvarenga, P. (2018). Contribuições da Análise do Comportamento para a compreensão da socialização emocional infantil. Acta Comportamentalia, 26(3), 379392. http://www.revistas.unam.mx/index.php/acom/article/view/66936

Lins, T. C. S. (2018). o impacto das práticas de socialização emocional sobre os problemas internalizantes na infância [Tese não publicada]. Universidade Federal da Bahia. https://www.repositorio.ufba.br/ri/handle/ri/26734

McCord, B. L., \& Raval, V. V. (2016). Asian indian immigrant and white american maternal emotion socialization and child socio-emotional functioning. Journal of Child and Family Studies, 25, 464-474. https://doi.org/10.1007/ s10826-015-0227-2

Mendes, M. G., \& Oliveira, G. F. (2013). Treine suas emoções, supere-se, seja feliz!: uma análise discursiva do imperativo da superação e da felicidade na literatura de autoajuda. PPGCOM - ESPM, Comunicação Mídia e Consumo, 10(29), 161-182. http://revistacmc.espm.br/index.php/revistacmc/article/view/601

Meyer, S., Raikes, H. A., Virmani, E. A., Waters, S., \& Thompson, R. A. (2014). Parent emotion representations and the socialization of emotion regulation in the family. International Journal of Behavioral Development, 38(2), 164-173. https:// doi.org/10.1177/0165025413519014 


\section{INTERACÃO EM LF PSICOLOGIA}

Miller-Slough, R., Zeman, J. L., Poon, J. A., \& Sanders, W. M. (2016). Children's maternal support-seeking: relations to maternal emotion socialization responses and children's emotion management. Journal of Child and Family Studies, 25, 3009-3021. https://doi.org/10.1007/s10826-016-0465-y

Mirabile, S. P. (2015). Ignoring children's emotions: a novel ignoring subscale for the coping with children's negative emotions scale. European Journal of Developmental Psychology, 12(4), 459-471. https://doi.org/10.1080/174056 29.2015.1037735

Morelen, D., \& Suveg, C. (2012). A Real-time analysis of paren$t$-child emotion discussions: the interaction is reciprocal. Journal of Family Psychology, 26(6), 998-1003. https://doi. org/10.1037/a0030148

Morelen, D., \& Thomassin, K (2013). Emotion Socialization and Ethnicity: an examination of practices and outcomes in african american, asian american, and latin american families. Yale Journal of Biology and Medicine, 86(2), 168-178. https://www.researchgate.net/publication/237843545_ Emotion_Socialization_and_Ethnicity_An_Examination_ of_Practices_and_Outcomes_in_African_American_Asian_ American_and_Latin_American_Families

Morelen, D., Shaffer, A., \& Suveg, C. (2014). Maternal emotion regulation: links to emotion parenting and child emotion regulation. Journal of Family Issues, 37(13), 1891-1916. https://doi.org/10.1177/0192513X14546720

Premo, J. E., \& Kiel, E. J. (2014). The effect of toddler emotion regulation on maternal emotion socialization: moderation by toddler gender. Emotion, 14(4), 782-793. https://doi. org/0.1037/a0036684

Rawana, J. S., Flett, G. L., McPhie, M. L. Nguyen, H. T., \& Norwood, S. J. (2014). Developmental trends in emotion regulation: a systematic review with implications for community mental health. Canadian Journal of Community Mental Health, 33(1). https://doi.org/10.7870/cjcmh-2014-004

Reis, A. H., Oliveira, S. E. S., Bandeira, D. R., Andrade, N. C., Abreu, N., \& Sperb, T. M. (2016). Emotion Regulation Checklist (ERC): Estudos preliminares da adaptação e validação para a cultura brasileira. Temas em Psicologia, 24(1), 77-96. https://doi.org/10.9788/tp2016.1-06

Robson, C. (1993) Real World Research: a resource for social scientists and practitioner researchers. Blackwell $\mathrm{Pu}-$ blishers Inc.
Rocha, A. M., Candeias, A. A., \& Silva, A. L. (2017). Regulação das emoções na infância: delimitação e definição. Psychologica, 61(1), 7-28. https://doi.org/10.14195/16478606_61-1_1

Rogers, M. L., Castro, V. L., Halberstadt, A. G., MacCormack, J. K., \& Garrett-Peters, P. (2016). Maternal emotion socialization differentially predicts third-grade children's emotion regulation and lability. Emotion, 16(2), 280-291. https:// doi.org/10.1037/emo0000142

Sabatier, C., Cervantes, D. R., Torres, M. M., Rios, O. H., \& Sañudo, J. P. (2017). Emotion regulation in children and adolescents: concepts, processes and influences. Psicología Desde El Caribe, 34(1). https://doi.org/10.14482/ psdc.34.1.9778

Shewark, E. A., \& Blandon, A. Y. (2015). Mothers' and fathers' emotion socialization and children's emotion regulation: a within-family model. Social Development, 24(2), 266-284. https://doi.org/10.1111/sode.12095

Shields, A. M., \& Cicchetti, D. (1995). The development of an emotion regulation assessment battery: reliability and validity among at-risk grade-school children [Paper presentation]. Biennial meeting of the society for research on child development, Indianapolis, IN, USA.

Souza, A. B. M., \& Mendes, D. M. L. F. (2018). Compreensão emocional em crianças e crenças maternas sobre competência emocional. Psicologia Clínica, 30(3), 541-559. https://doi.org/10.33208/PC1980-5438v0030n03A07

Verma, G. K., \& Tiwary, U. S. (2014). Multimodal fusion framework: a multiresolution approach for emotion classification and recognition from physiological signals. Neurolmage, 102, 162-172. https://doi.org/10.1016/j.neuroimage.2013.11.007

Whitebread. D., \& Basilio, M. (2012). The emergence and early development of self-regulation in young children. Professorado, 16(1). https://www.ugr.es/ recfpro/rev161ART2en.pdf

Zeman, J., Cassano, M., Perry-Parrish, C., \& Stegall, S. (2006). Emotion regulation in children and adolescents. Developmental and Behavioral Pediatrics, 27(2). https://doi. org/10.1097/00004703-200604000-0001

Data da Submissão: 05/08/2020 Primeira decisão editorial: 05/11/2020 Aceite: $18 / 11 / 2020$ 